\title{
Genes Involved in the Uptake and Catabolism of Gluconate by Escherichia coli
}

\author{
By BRIGITTE BÄCHI* AND H. L. KORNBERG† \\ Department of Biochemistry, School of Biological Sciences, \\ University of Leicester, Leicester $L_{\mathrm{I}}{ }_{7} \mathrm{RH}$
}

(Received I6 May 1975)

\begin{abstract}
SUMMARY
The isolation and properties of a mutant of Escherichia coli $\mathrm{KI} 2$ that is totally unable to take up and utilize gluconate are described. Genetical analysis shows this phenotype to be associated with two lesions. One phenotype, designated $\mathrm{GntM}^{-}$, is the result of a mutation in a gene co-transducible with malA; the other, designated GntS-, is the result of a mutation in a gene (gntS) co-transducible with $f d p$. The GntS--phenotype differs little from that of wild-type cells, but GntM- GntS ${ }^{+}$ organisms grow on gluconate only after a prolonged lag and form a gluconate uptake system that is strongly repressed by pyruvate. Moreover, such GntM- mutants readily give rise to further mutants that form a gluconate uptake system, gluconate kinase and 6-phosphogluconate dehydratase constitutively; in partial diploids, this constitutivity is recessive to the inducible character. It is postulated that the $\mathrm{GntM}^{-}$ phenotype is due to malfunction of a negative control gene gnt $R$, and that gnt $S^{+}$ specifies the activity of a gluconate uptake system.
\end{abstract}

\section{INTRODUCTION}

Two steps must precede the entry of gluconate into the main metabolic routes that effect its utilization by Escherichia coli. In the first step, gluconate present externally must enter the cell. In $E$. coli, this entry occurs by an 'active transport' process, in which metabolic energy is transduced into a vectorial movement of the substrate (in the form of gluconic acid, or as the gluconate ion either accompanied by $\mathrm{H}^{+}$or balanced by counter-transport of $\mathrm{OH}^{-}$; Robin \& Kepes, 1973). In the second step, the gluconate thus taken up is phosphorylated to 6-phosphogluconate, with concomitant conversion of ATP to ADP; this reaction is catalysed by gluconate kinase (EC. 2.7.I . 12; Cohen, 1951).

Although 6-phosphogluconate is readily formed from glucose-6-phosphate by a constitutive enzyme in $E$. coli (Fraenkel \& Banerjee, 1972), the two steps leading to its formation from external gluconate would not need to occur unless gluconate were present in the external milieu. Indeed, both the uptake system and the kinase are induced in response to gluconate (Eisenberg \& Dobrogosz, 1967) though neither is induced by internally-generated 6-phosphogluconate (Kornberg \& Soutar, 1973). Mutants have been described that form the gluconate uptake system, gluconate kinase and 6-phosphogluconate dehydratase (EC. 4.2.1 . I2) in high activity in the absence of gluconate, which shows that the structural genes that specify these proteins are under the control of a regulatory element. Zwaig et al.

* Present address: Abteilung Mikrobiologie, Biozentrum der Universität Basel, Klingelbergstrasse 70, CH-4056 Basel, Switzerland.

$\uparrow$ Present address: Department of Biochemistry, Tennis Court Road, The University, Cambridge CB2 IQW. 
(1973) have located this regulatory gene, designated gnt $R$, at about minute 66 on the $E$. coli linkage map (Taylor \& Trotter, 1972), co-transducible with the malA and asd markers.

These constitutive mutants were derived from a mutant (designated M2) which grew on gluconate only after a long lag; a gluconate uptake system, gluconate kinase and 6-phosphogluconate dehydratase were inducibly formed at specific activities lower than those observed with wild-type cells. This behaviour accords with the postulated dysfunction of a regulatory gene. However, two other mutants, in which chromosomal markers including malA and asd were deleted, exhibited neither the ability to take up gluconate nor to phosphorylate it, and yet formed 6-phosphogluconate dehydratase constitutively; it is therefore likely that structural genes for the two missing functions also lie in this region of the chromosome (Nagel de Zwaig et al. 1973). Indeed, a gene specifying an uptake system for gluconate, designated $u s g A$, was found to be highly co-transducible with malA (Faik \& Kornberg, 1973). However, like the mutant M2 described by Nagel de Zwaig et al. (1973), usg A mutants grow on gluconate after long lag with gradual acceleration of growth rate; under these conditions, they form a system for gluconate uptake, gluconate kinase and 6-phospho-2-keto-3deoxygluconate aldolase (EC. 4.1.2. I4) at specific activities lower than observed with their parents. This behaviour is not the consequence of further mutation, but indicates the induction of a second system that gradually assumes the functions of the impaired first system.

The existence of such a second system was suggested also by the properties of a mutant R6, which was impaired in gluconate kinase and devoid of 6-phosphogluconate dehydrogenase (gnd) EC. I . I I . 43) and 6-phosphogluconate dehydratase (edd) activities but which retained the ability to take up (and accumulate) gluconate (Pouysségur, Faik \& Kornberg, 1974). When exposed to gluconate for a prolonged period, some gluconate kinase activity was induced; similarly, when transduced to gnd $^{+}$and $e d d^{+}$, the organisms grew on gluconate, albeit initially slowly but with gradual acceleration (P. Faik and H. L. Kornberg, unpublished experiments). It is possible that a second form of gluconate kinase, as reported briefly by Hung, Orozco \& Zwaig (1970), was being induced under these conditions.

It is the main purpose of this paper to report the properties of mutants impaired in such a second set of genes specifying activities of a gluconate uptake system and/or a gluconate kinase. A double mutant of $E$. coli was isolated that is unable to grow on gluconate because it is impaired in two components of the complement of genes that specify the utilization of this substance. One dysfunction is in the regulation of that set bearing the main responsibility for gluconate utilization; the phenotype of organisms thus affected is designated $\mathrm{GntM}^{-}$for convenience. The other dysfunction is in the activity of a subsidiary set, designated gntS. The genes affected have been transferred separately to suitable recipients. The lesion in $\mathrm{GntM}^{-}$ mutants, that maps at about minute 66 , is probably identical to that in the gntM2 mutant that is affected in a regulatory element $g h t R$ and that has been described by Nagel de Zwaig et al. (1973); gnt $S$ mutants, which map at about minute 85 (co-transducible with $f d p$ ), have not previously been described.

\section{METHODS}

Organisms. Strains of $E$. coli used, and their provenance, are listed in Table I. Those prepared in the course of this work carry the prefix ' $\mathrm{BB}$ '; their selection is described in the text.

Growth and analysis of bacteria. The composition of media, the conditions used for cell culture, and the procedures employed for measurements of cell density, gluconate uptake and gluconate kinase activity, were those recently described (Bächi \& Kornberg, 1975).

Units. The specific rates of gluconate uptake by washed cells incubated with $0.05 \mathrm{mM}-$ 
Table I. Organisms used in this work

The genetic markers used are those listed by Taylor \& Trotter (1972), with the exception of usg $A$ (Faik \& Kornberg, 1973), and of $g n t R^{+}, g n t R \mathrm{I}, g n t R$ and $g n t S$, which accord with the notation suggested by Zwaig et al. (1973) and are defined in the text.

\begin{tabular}{|c|c|}
\hline Strain & Genotype \\
\hline \multicolumn{2}{|l|}{ Hfr strains } \\
\hline KI . I & metB thy pps \\
\hline $\mathrm{KI} \cdot \mathrm{I} \cdot 2 \cdot 5^{\mathrm{c}}$ & met B thy pps ppc iclR \\
\hline KI I. I I $2 \mathrm{~h}$ & met B thy pps gntRI gntS \\
\hline PIO & thr leu lac Y malB \\
\hline PFI & met $B$ usg $A$ \\
\hline PFia & $\arg H$ usg $A$ \\
\hline PI 3 & his cys pro trp gal \\
\hline KIO.I9 & $f d p$ \\
\hline $\begin{array}{l}\text { RE37 } \\
\text { KL1699 }\end{array}$ & $\begin{array}{l}\text { met } B \text { pyrE uhp } \\
\operatorname{rec} A\end{array}$ \\
\hline \multicolumn{2}{|l|}{$\mathrm{F}^{-}$strains } \\
\hline K2.It & his argH thr leu malA xyl pps str \\
\hline BB I & metB his thr leu malA xyl gntRI gntS pps str \\
\hline BB 12 & met $B$ his thr leu malA xyl gntR gntS pps str \\
\hline BB52 & his thr leu malA xyl gntRI gntSpps fdp str \\
\hline BB54 & his thr leu malA xyl gntRI pps fdp str \\
\hline BB60 & his thr leu malA xyl gntRI pps str \\
\hline BB63 & his thr leu xyl gntS pps fdp str \\
\hline BB73 & his thr leu xyl pps str \\
\hline BB77 & met $B$ thr leu malA xyl gnt $R$ gntS pps recA str \\
\hline BEI 38 & his thr leu malA xyl gntRI str \\
\hline \multicolumn{2}{|l|}{$\mathbf{F}^{\prime}$ merozygotes } \\
\hline MAFI/JCI 553 & $\begin{array}{l}\mathrm{F}^{\prime} 140\left(\mathrm{fda}^{+} \mathrm{mtl}^{+}\right) / \arg A \text { met } B \text { his leu mtl rec } A \\
x y l \text { malA gal lac } Y \text { tonA supE }\end{array}$ \\
\hline KLF4I/JCI 553 & $\begin{array}{l}\mathrm{F}^{\prime} \text { I4I }\left(\text { asd } d^{+} \text {arg } G^{+}\right) / \arg G \text { metB his leu mtl rec } A \\
x y \text { malA gal lac } Y \text { tonA supE }\end{array}$ \\
\hline
\end{tabular}

Source or reference

Brice \& Kornberg (1967)

Kornberg \& Smith (1969)

This paper

Gift from F. Jacob

Faik \& Kornberg (1973)

$\mathrm{Met}^{+} \mathrm{Arg}^{-}$derivative of PFI

Gift from F. Jacob

Gift from R. A. Cooper

Essenberg \& Kornberg (1975)

Gift from B. Bachmann

Brice \& Kornberg (1967)

This paper

[Revertant of BBI]Gluconate ${ }^{+}$

[Kro.19× BBI] $\mathrm{Met}^{+} \mathrm{Str}^{\mathrm{R}}$

[KIO.19 $\times$ BBI] $\mathrm{Met}^{+}$Str $^{\mathrm{R}}$

[PI(PFIa) $\times$ BB52]Fdp ${ }^{+}$

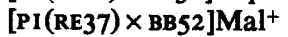

[PI(RE37) $\times$ BB6o]Mal $^{+}$

[KLI $699 \times$ BBI 2] His ${ }^{+}$Str $^{R}$

[PI(KI, .19) $\times$ BB60]Pps ${ }^{+}$

Gift from B. Bachmann

Gift from B. Bachmann

$\left[{ }^{14} \mathrm{C}\right]$ gluconate at $25{ }^{\circ} \mathrm{C}$ were calculated from the initial rates measured over the first ro $\mathrm{s}$ after addition of the isotopic material; they are here expressed as nmol gluconate taken up/ $\mathrm{min} / \mathrm{mg}$ dry mass of cells. The specific activities of gluconate kinase in sonic extracts of the organisms are expressed as nmol gluconate phosphorylated at $25^{\circ} \mathrm{C} / \mathrm{min} / \mathrm{mg}$ protein.

Genetical procedures. The procedures used for the location of genes by periodic interruption of conjugation and by transduction with phage PIkc were those described by Brice \& Kornberg (1967). Other techniques, such as those used for the construction and identification of strains carrying the $\operatorname{rec} A$ marker and the construction of partial diploids, were those compiled by Miller (1972). All partial diploids were maintained on selective media; they were checked for their ability to act as genetic donors and to transfer the episomal gluconate alleles, and were tested for spontaneous segregation of cells with the predicted haploid phenotype.

Chemicals. Sodium [U-14C]gluconate was purchased from the Radiochemical Centre, Amersham, Buckinghamshire. 6-Phosphogluconate dehydrogenase, ATP and NADP were bought from Boehringer Corp. (London) Ltd. All other reagents were of the highest purity readily available commercially. 


\section{RESULTS AND DISCUSSION}

\section{Isolation of a mutant affected in early steps of gluconate catabolism}

Mutants impaired in the catabolism of carbohydrates can readily be obtained by the method of Kornberg \& Smith (1969). In this procedure, use is made of the ability of substances catabolized to phosphoenolpyruvate (PEP) or pyruvate to inhibit the growth on acetate of strains of $E$. coli devoid of PEP-carboxylase activity ( $p p c$; EC. 4. I . I . 3I); further mutants that now tolerate the previously inhibitory substance either no longer take it up or are impaired in its catabolism.

When $E$. coli strain KI I.2.5 was thus plated on media containing both sodium acetate $(20 \mathrm{mM})$ and sodium gluconate $(5 \mathrm{mM})$ as carbon sources, isolated colonies appeared after 2 to 3 days at $37^{\circ} \mathrm{C}$. These colonies were picked and purified by repeated isolation of single colonies. They were found to be still devoid of PEP-carboxylase activity, and would therefore not grow on any hexose or other substrate which requires this anaplerotic enzyme for catabolism, until the $p p c^{+}$allele had been introduced. This was done by infecting suspensions of the organisms with phage PIkc that had been grown on the isogenic $p p c^{+}$strain KI.I (Brice \& Kornberg, 1967); the Ppc ${ }^{+}$transductants were selected on plates containing glucose6-phosphate as sole carbon source. Among the $\mathrm{Ppc}^{+}$transductants obtained from a number of such initially gluconate-tolerant $p p c$ mutants was one (designated KI . I . I 2h) that did not grow on plates containing gluconate as sole carbon source over several days at $37{ }^{\circ} \mathrm{C}$, although growth on plates containing glucose, glucose-6-phosphate, glucuronate or galacturonate was rapid. Washed suspensions of this organism, grown on nutrient broth or glycerol in the presence of gluconate, took up isotope from sodium $\left[\mathrm{U}-{ }^{14} \mathrm{C}\right]$ gluconate at only very low rates, and cell-free extracts were devoid of gluconate kinase activity. In contrast, other $\mathrm{Ppc}^{+}$transductants from the initially gluconate-tolerant $p p c$ mutants, whose growth on a variety of hexoses and hexuronic acids had also been restored, grew slowly on gluconate; gluconate uptake and gluconate kinase formation were induced in such mutants, and their properties are not considered further.

It was thus likely that the mutant KI . I . I $2 \mathrm{~h}$ was impaired in more than one function. The unknown lesion(s) preventing its growth on gluconate were transferred by conjugation into the genetic recipient strain $\mathrm{K2}$. It. $\mathrm{Arg}^{+} \mathrm{Str}^{\mathrm{R}}$ recombinants were selected on glucose medium also containing methionine, and these were screened for any that had lost their ability to grow on gluconate. Strain BBI, which was unable to grow on gluconate, was thus obtained and was used for further biochemical and genetic studies. This strain grows at wild-type rates on $\mathrm{C}_{6}$ sources like glucose, fructose or glucuronate, and on the $\mathrm{C}_{3}$ source, glycerol. It grows very slowly on gluconate, with a doubling time of over $10 \mathrm{~h}$, and appears neither to be able to induce its gluconate uptake system above basal levels nor to form gluconate kinase in significant activity (Table 2 ).

\section{Location of the genes affected on the chromosome of strain $\mathrm{BB} \mathrm{I}$}

By using a variety of $\mathrm{Hfr}$ strains that transfer their genetic material to the $\mathrm{F}^{-}$recipient BBI from different points of origin, the markers that prevented the growth of this strain on gluconate as sole carbon source could be located on its chromosome. The Hfr strains used, and the origin and directions of their gene transfer, are shown in Fig. I.

Conjugation of the Hfr strain PIO and strain BBI gave recombinants, selected either for $\mathrm{Met}^{+} \mathrm{Str}^{\mathrm{R}}$ or for $\mathrm{Mal}^{+} \mathrm{Str}^{\mathrm{R}}$, with apparently wild-type phenotype on gluconate. Measurements of the time of entry of the gene which confers the ability on strain BBI to grow on 


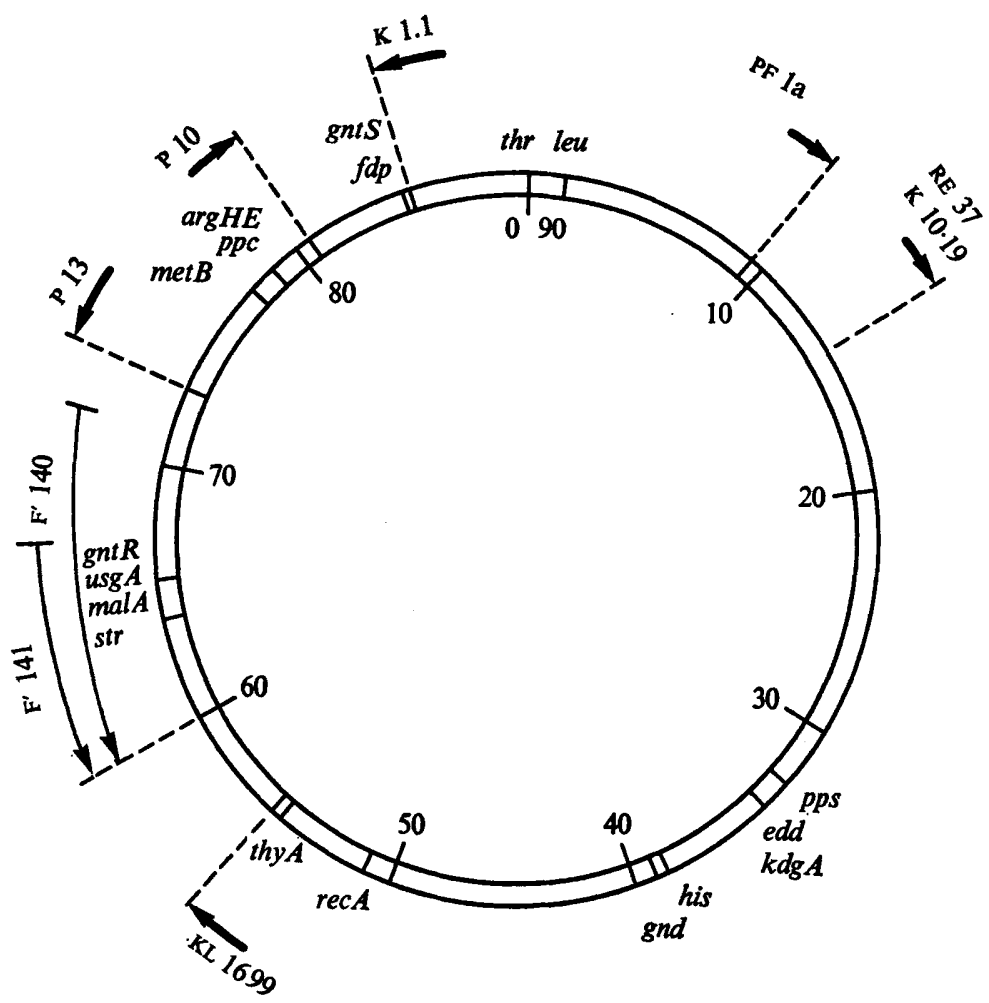

Fig. I. Linkage map of $E$. coli, showing the location of relevant markers, and the origins and directions of genome transfer of $\mathrm{F}^{\prime}$ episomes and $\mathrm{Hfr}$ strains used.

gluconate, by periodic interruption of conjugation, showed it to be located at about minute 66 on the $E$. coli linkage map, close to malA. As shown later, this restoration of growth on gluconate $\mathrm{GntM}^{+}$is due to the re-introduction of a functional regulator gene $\left(g n t R^{+}\right)$into the mutant BBI impaired in it. This impairment of the $g n t R$ gene is here designated gnt $R \mathrm{I}$.

On gluconate tetrazolium plates, $\mathrm{GntM}^{+}$recombinants gave white colonies. The growth curve and gluconate uptake curves, of induced and uninduced cultures, of one such $\mathrm{GntM}^{+}$ gnt $S$-recombinant are shown in Fig. $2 b$.

By mating the $\mathrm{F}^{-}$mutant $\mathrm{BBI}$ with the Hfr strains PI3, PFIa and $\mathrm{KI}$. I, and selecting recombinants for MetB ${ }^{+} \operatorname{Str}^{\mathbb{R}}$ (PI3), Thr $^{+}$Leu $^{+}$Str $^{\mathrm{R}}$ (PFIa) and $\mathrm{Thr}^{+} \mathrm{Leu}^{+} \mathrm{Str}^{\mathrm{R}}$ (KI.I) respectively, a second marker, mapping between minutes 84 and 86 on the linkage map, was found to restore growth on gluconate plates. For convenience, this marker will be designated gntS. The GntS ${ }^{+}$recombinants thus obtained were still red on gluconate tetrazolium plates and hence were still GntM-. Recombinants of this type showed a long lag before growing on gluconate but their subsequent growth occurred at wild-type rate (Fig. 2c). In further contrast to $\mathrm{GntM}^{+}$organisms, there was very little induction of the gluconate uptake system by the $\mathrm{GntM}^{-} \mathrm{GntS}^{+}$recombinants when gluconate was added to cultures growing on another carbon source (e.g. fructose); however, the organisms grown on gluconate as sole carbon source attained gluconate uptake activities as high or even higher than achieved by the similarly-grown cultures of the parental strain K2. It (Fig. $2 a, c$ ). As a control, a cross of the original strain KI . I . I2h with the $\mathrm{GntM}^{-} \mathrm{GntS} \mathrm{S}^{+}$mutant BB2 I was performed, selecting for $\mathrm{Thr}^{+} \mathrm{Leu}^{+} \mathrm{Str}^{\mathrm{R}}$. Twenty-one out of $\mathrm{I}$ Io such recombinants obtained were unable to grow on 


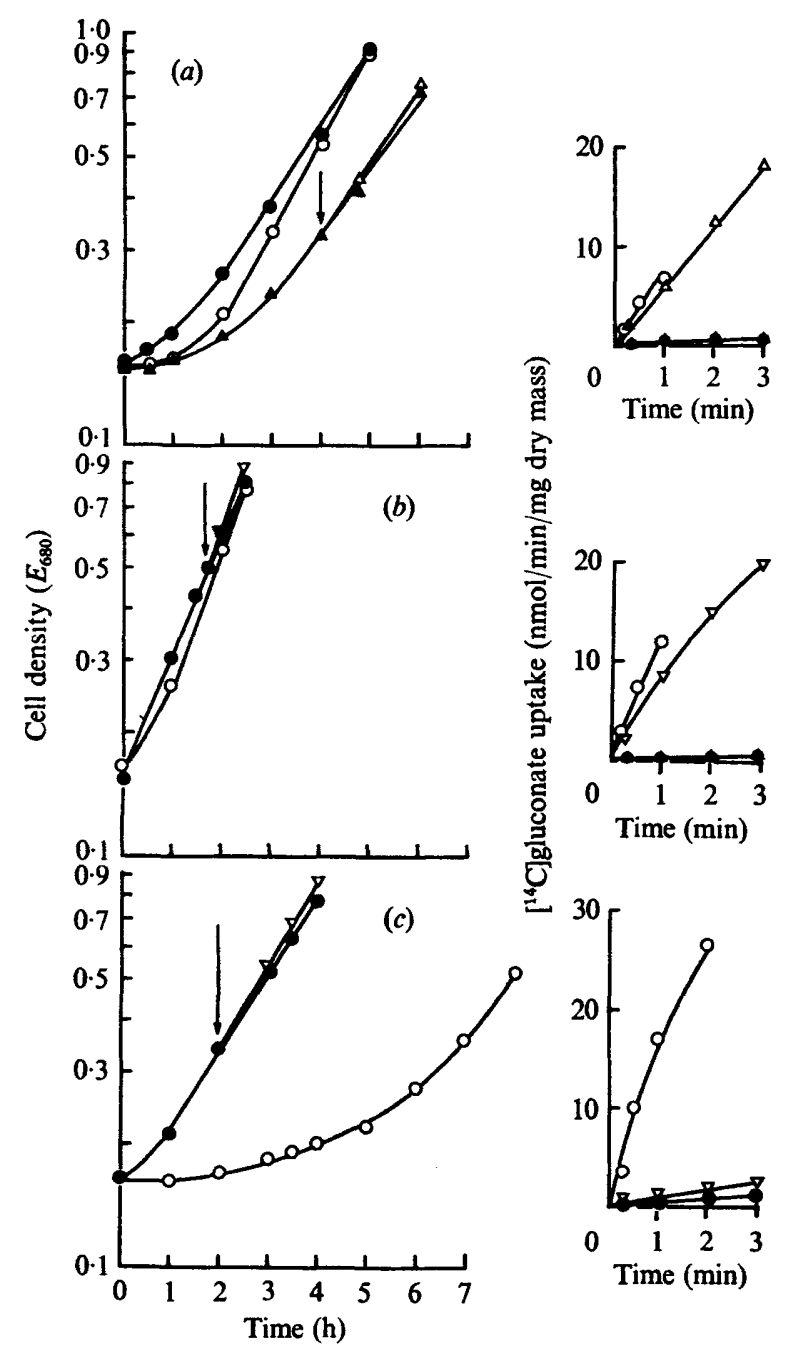

Fig. 2. Left-hand side: growth of $(a)$ glycerol-grown strain $\mathrm{K2}$.1t $\left(\mathrm{GntM}^{+} \mathrm{Gnt} \mathrm{S}^{+}\right)$, (b) fructosegrown strain BB35 $\left(\mathrm{GntM}^{+} \mathrm{GntS}^{-}\right)$, and $(c)$ fructose-grown strain BB2I $\left(\mathrm{GntM}^{-} \mathrm{GntS}^{+}\right)$on $(\bullet)$ fructose, $(O)$ gluconate, and $(\Delta)$ glycerol. At the points marked with arrows gluconate was added to duplicate fasks of $(\triangle)$ strain $\mathbf{2 2}$. It growing on glycerol and $(\nabla)$ strains BB35 and 21 growing on fructose. Right-hand side: samples of the cells thus grown were harvested, washed, and the rates at which they took up $0.05 \mathrm{~mm}-\left[{ }^{14} \mathrm{C}\right]$ gluconate were measured.

gluconate and had therefore acquired the gnt $S$ allele of the donor strain. Since the location of gnt $S$, by interrupted conjugation, at about minute 85 , suggested its proximity to the $f d p$ gene that specifies fructose I,6-bisphosphatase activity (EC. 3.I.3.I I; Yu, Kaney \& Atwood, 1965), recombinants were selected on glucose for $\mathrm{Met}^{+} \mathrm{Str}^{\mathrm{R}}$ from the cross [KIO. I9 (HfrC, $f d p) \times \mathrm{BBI}$ ]. These recombinants were screened for their ability to grow on glycerol: those that did not grow on this $\mathrm{C}_{3}$ compound had received the $f d p$ allele. Among these $f d p$ recombinants was one (BB52) that was still totally unable to grow on gluconate and that presumably had retained both the gnt $S$ marker as well as that responsible for the GntMphenotype. Another (BB54) grew on gluconate but gave deep red colonies on gluconate- 
Table 2. Induction of gluconate uptake and gluconate kinase activities in strain $\mathrm{K2}$. It and the mutant BBI

The strains were grown on $20 \mathrm{~mm}$-glycerol overnight and were then allowed to grow for 2 to 3 doublings on the carbon sources indicated. Gluconate uptake and gluconate kinase activities were measured as described in Methods.

\begin{tabular}{cc} 
Strain & \multicolumn{1}{c}{ Carbon source } \\
K2.It & $20 \mathrm{~mm}$-Glycerol \\
& $20 \mathrm{~mm}$-Glycerol + $10 \mathrm{mM}$-gluconate \\
BBI & $20 \mathrm{~mm}$-Glycerol \\
& $20 \mathrm{~mm}$-Glycerol + $10 \mathrm{~mm}$-gluconate
\end{tabular}

$\overbrace{\begin{array}{c}\text { Gluconate uptake } \\ 0.4\end{array}}^{\text {Specific activity of }} \begin{array}{cr}\text { Gluconate kinase } \\ 7.0 & 13 \\ 0.8 & 268 \\ 1.2 & 9 \\ & 14\end{array}$

tetrazolium plates: it had clearly acquired the gnt $S^{+}$allele of the genetic donor but had remained $\mathrm{GntM}^{-}$. Both these recombinants were also unable to grow on maltose.

The availability of these $f d p$ mutants permitted the more precise location, by phage PI kc-mediated transduction, of the gnt $S$ marker on the $E$. coli chromosome.

When the phage was propagated on the Hfr strain PFIa which is impaired in the gluconate uptake system specified by the usg $A$ gene located at about minute 66 on the linkage map (Faik \& Kornberg, 1973), and this phage was used to infect the $f d p$ malA recipient BB52 that was phenotypically both $\mathrm{GntM}^{-}$and $\mathrm{GntS}^{-}, 40$ out of $123 \mathrm{Fdp}^{+}$transductants were of the GntM- GntS ${ }^{+}$phenotype; the gntS marker is thus about $33 \%$ co-transducible with $f d p$. One such transductant (вв60) was used for a second transduction. In this, phage propagated on the HfrC derivative RE37 of E. coli KI2 (Essenberg \& Kornberg, 1975), which is mal $A^{+}$, was used to infect $\mathrm{BB} 60$; of $5 \mathrm{I} \mathrm{Mal}+$ transductants tested, 23 were $\mathrm{GntM}^{+}$. Similarly, screening of $\mathrm{Mal}^{+}$transductants obtained after infection of strains BB52 (GntM- ${ }^{-}$GntS $^{-}$) and BB54 $\left(\mathrm{GntM}^{-} \mathrm{GntS}^{+}\right)$with this phage stock showed the gene $\left(g n t R^{+}\right)$that restored the $\mathrm{GntM}^{+}$ phenotype to be over $60 \%$ co-transducible with"malA.

\section{Gluconate kinase and gluconate uptake activities in mutants affected in GntM and GntS activities}

In the extreme case, mutants (such as strain BBI) which are impaired both in the allele specified by $g n t R^{+}$and that specified by $g n t S^{+}$, neither take up gluconate nor form gluconate kinase when exposed to gluconate, whereas wild-type cells, readily inducible for both activities, take up about $20 \mathrm{nmol}$ of gluconate/min/mg dry mass and phosphorylate about $460 \mathrm{nmol}$ gluconate/min/mg protein (Table 2). Restoration of $g n t R^{+}$by the transduction of this marker into strain BBI yields progeny, the properties of which do not differ significantly from those of the wild-type organism. On the other hand, the introduction of gnt $S^{+}$into the double mutant BBI gives progeny (such as the strain BB2I shown in Fig. 2c) that grow on gluconate only after a prolonged lag on transfer to medium containing this substance as sole carbon source. The length of this lag is inversely proportional to the gluconate concentration in this medium. Thus a fructose-grown culture of strain BB2I took $3.2 \mathrm{~h}$ before the full exponential rate of growth was obtained on $10 \mathrm{mM}$-gluconate, but less than $2 \mathrm{~h}$ when $50 \mathrm{mM}$-gluconate was used; intermediate concentrations sustained exponential growth after lags intermediate between these times. Even after the attainment of exponential growth on gluconate, the gluconate kinase activity of extracts of GntM- mutants is less than half of that of $\mathrm{GntM}^{+}$cells, and this is not increased however long the cells grow on gluconate. The gluconate uptake activity, however, approximates to that of wild-type cells. 
It thus appears that the introduction of the $\mathrm{GntS}^{+}$character into $\mathrm{GntM}^{-} \mathrm{GntS}^{-}$mutants restores fully the ability of the cells to take up gluconate, but that the ability to form gluconate kinase (in response to the gluconate taken up) is still impaired by the absence of GntM function. It may thus be that gntS specifies the activity of a gluconate uptake system. Since it is known (Nagel de Zwaig et al. 1973; Zwaig et al. 1973) that the activity of the uptake system specified by the usg $A$ marker (Faik \& Kornberg 1973) is governed by gene(s) co-transducible with malA (located at minute 66) and hence far removed from the gntS marker (located at minute 85 ), it must further be assumed that these uptake systems are different in properties, in regulation, or in both.

A major difference in the manner of their cellular regulation was indeed revealed by the effect of pyruvate on their synthesis. It is known that the growth of $E$. coli mutants devoid of PEP-synthetase activity (pps) on sugars taken up via the PEP-phosphotransferase system (Roseman, 1969) is strongly inhibited by added pyruvate, but that growth on substances taken up by active transport, such as gluconate, is little affected by pyruvate (Morgan \& Kornberg, 1969). This is found to hold also for pps mutants that lack the system specified by gnt $S^{+}$and that utilize gluconate via the GntM system. As shown in Fig. $3 a$, the addition of Io mM-pyruvate to a culture of such a gntS pps strain, growing on gluconate, retarded growth only slightly; there was only a slight reduction in the rates at which the gluconate uptake system (Fig. $3 b$ ) and gluconate kinase (Fig. $3 c$ ) continued to be synthesized. Similarly, the adaptation of a fructose-grown culture of this mutant to growth on gluconate plus pyruvate occurred as readily as when pyruvate was omitted, and growth on the former medium was only slightly slower than on the latter (Fig. 4a); the rates at which the gluconate uptake system and gluconate kinase activities continued to be formed under these conditions did not differ greatly (Fig. $4 b, c$ ). This behaviour of gnt $S$ mutants appeared to be identical to that of the otherwise isogenic pps mutant BB73 that carries both $\mathrm{GntM}^{+}$and $\mathrm{GntS}^{+}$activities (Fig. 5).

In contrast, $\mathrm{GntS}^{+}$mutants that are phenotypically $\mathrm{GntM}^{-}$, and which thus can adapt to growth on gluconate as sole carbon source either in liquid culture or on solid media, do not do so even after a week at $37^{\circ} \mathrm{C}$ if pyruvate is also present. Moreover, the addition of pyruvate to cultures of such mutants growing on gluconate results in progressive retardation of growth and a virtually complete cessation of synthesis both of the gluconate uptake system and of gluconate kinase. The introduction of the $p p^{+}$allele, by phage-mediated transduction into such pps mutants, did not alter this behaviour. Like extracts of its pps parent BB60, extracts of the $p p s^{+}$transductant $\mathrm{BBI}^{38}$, that was still $\mathrm{GntM}^{-}$, contained gluconate kinase at less than half of the specific activity found in extracts of $\mathrm{GntM}^{+}$cells after prolonged growth on gluconate (Table 3). Addition of pyruvate to a culture of this organism growing on gluconate did not significantly inhibit growth (Fig. 6a) but led to a virtually complete repression of the synthesis of the gluconate uptake system and of gluconate kinase (Fig. 6b,c). Neither of these activities was induced when fructose-grown cells were placed on medium containing both gluconate and pyruvate, although the $p s^{+}$mutant grew readily under these conditions and, in the absence of pyruvate, inducibly formed both the uptake system and the kinase (Fig. 7).

Although these results do not provide conclusive evidence that pyruvate is the metabolic repressor of the synthesis of the component(s) specified by $g n t S^{+}$, some support for such a conclusion is provided by the unusually large effect exerted by pyruvate, in comparison with other metabolites, when added to cultures of the GntM- mutant BBr 38 (Table 4). The high specific activities of the uptake system observed after growth on gluconate were lowered by the simultaneous provision of a number of precursors of pyruvate, but not by proline or 

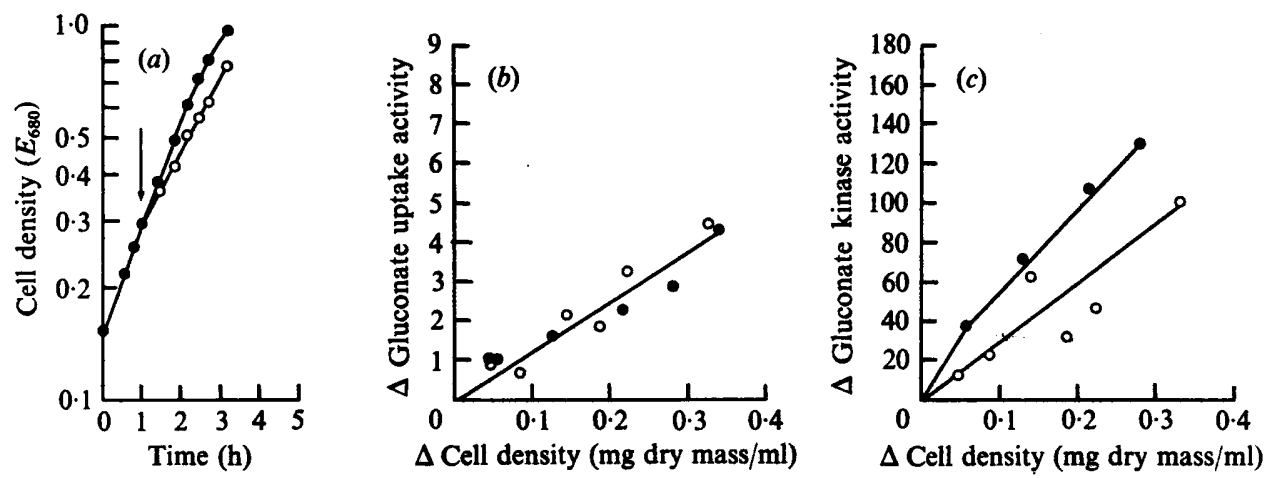

Fig. 3. The growth (a), the uptake of gluconate $(b)$, and the activity of gluconate kinase (c), of gluconate-grown strain BB63 (GntM+ ${ }^{+} \mathrm{GntS}^{-} \mathrm{Pps}^{-}$) continuing to grow on (O) 10 mM-gluconate or (after the point indicated by the arrow) on (O) $10 \mathrm{~mm}$-gluconate + $10 \mathrm{~mm}$-pyruvate. Samples of the growing cultures were harvested at various times, washed and the rates at which they took up $0.05 \mathrm{mM}-\left[{ }^{4} \mathrm{C}\right]$ gluconate were measured; other samples were disrupted by sonic oscillation for the measurement of gluconate kinase activity. The differential rates of synthesis (Monod, 1956) of gluconate uptake and gluconate kinase are plotted as the increases in total activity ( $\triangle$ specific activity $\times$ cell density) against the increases in cell density.
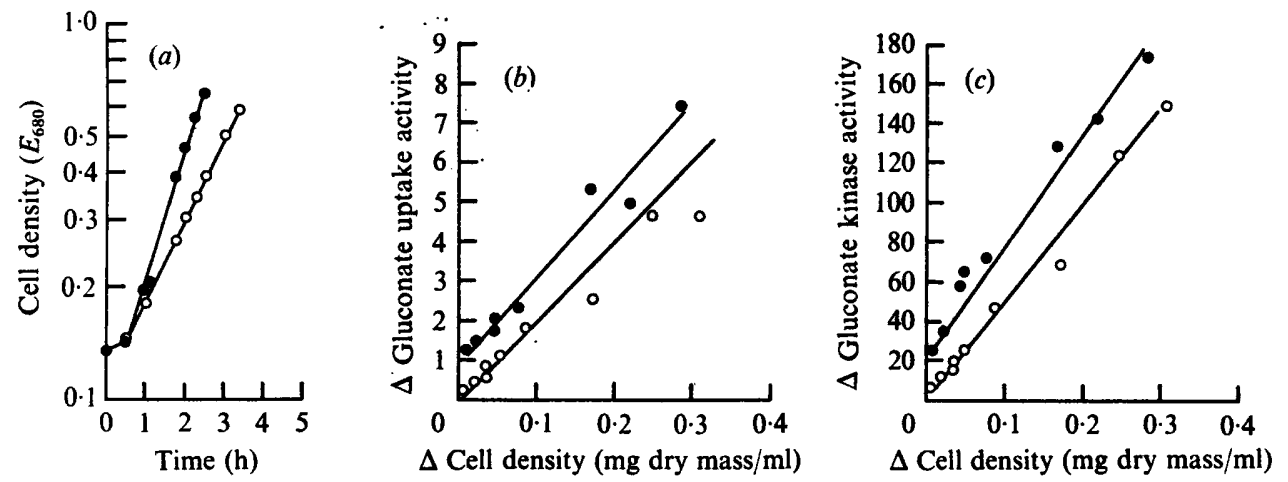

Fig. 4. The growth $(a)$, the uptake of gluconate $(b)$, and the activity of gluconate kinase $(c)$, of fructose-grown strain BB63 (GntM+ ${ }^{+}$GntS- Pps $^{-}$) adapting to growth on (O) 10 mM-gluconate, or (O) 10 mM-gluconate + 10 mM-pyruvate. Samples were treated as described for Fig. 3.
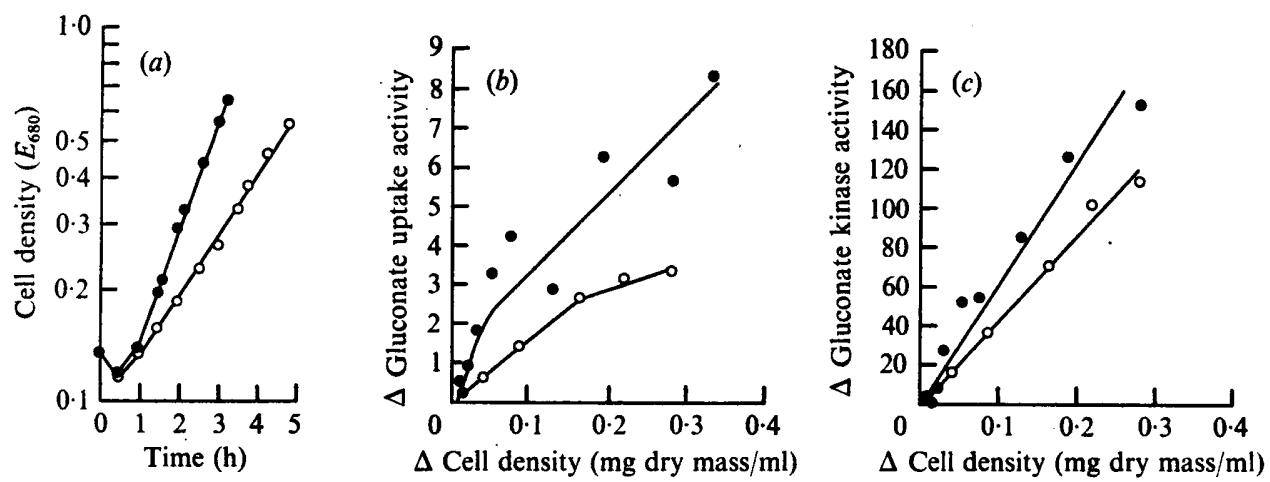

Fig. 5. The growth $(a)$, the uptake of gluconate $(b)$, and the activity of gluconate kinase $(c)$, of fructose-grown strain BB73 $\left(\mathrm{GntM}^{+} \mathrm{GntS}^{+} \mathrm{Pps}^{-}\right.$) adapting to growth on (O) Io mM-gluconate or (O) 10 mM-gluconate + Io mM-pyruvate. Samples were treated as described for Fig. 3. 
Table 3. Specific activities of gluconate uptake and gluconate kinase of different mutants in gluconate metabolism

The strains BB73, Bв63, Bв60 and BBI 38, isogenic except for the genetic markers mentioned below and such other genes co-transduced by phage $\mathrm{PI}$, were grown on $10 \mathrm{mM}$-gluconate. The rates of gluconate uptake and the activity of gluconate kinase were determined as described in Methods.

\begin{tabular}{|c|c|c|c|}
\hline \multirow[b]{2}{*}{ Strain } & \multirow[b]{2}{*}{ Genotype } & \multicolumn{2}{|c|}{ Specific activity of } \\
\hline & & Gluconate uptake & Gluconate kinase \\
\hline BB73 & $g n t R^{+} g n t S^{+} p p s$ & 22 & 465 \\
\hline B863 & $g n t R^{+} g n t S p p s$ & 20 & 490 \\
\hline BB60 & $g n t R I g n t S^{+} p p s$ & 25 & 194 \\
\hline BBI 38 & gntRI $g_{n t S^{+}} p s^{+}$ & 25 & 163 \\
\hline
\end{tabular}
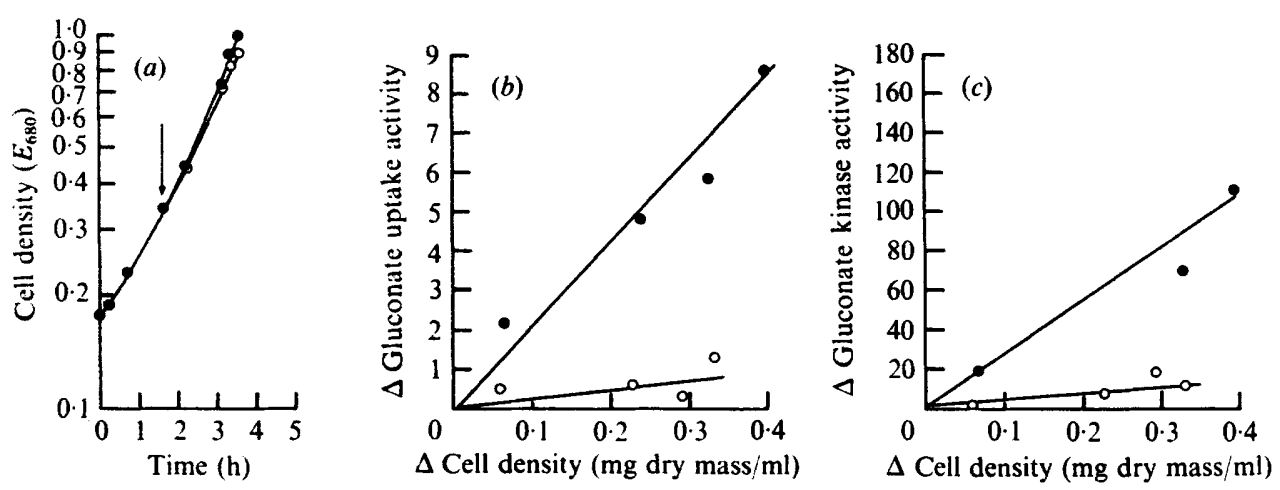

Fig. 6. The growth $(a)$, the uptake of gluconate $(b)$, and the activity of gluconate kinase $(c)$, of gluconate-grown strain BBI $38\left(\mathrm{GntM}^{-} \mathrm{GntS}^{+}\right.$) continuing to grow on (O) IO mM-gluconate or (after the point indicated by the arrow) on $(\mathrm{O})$ Io mM-gluconate + IO mM-pyruvate. Samples were treated as described for Fig. 3.
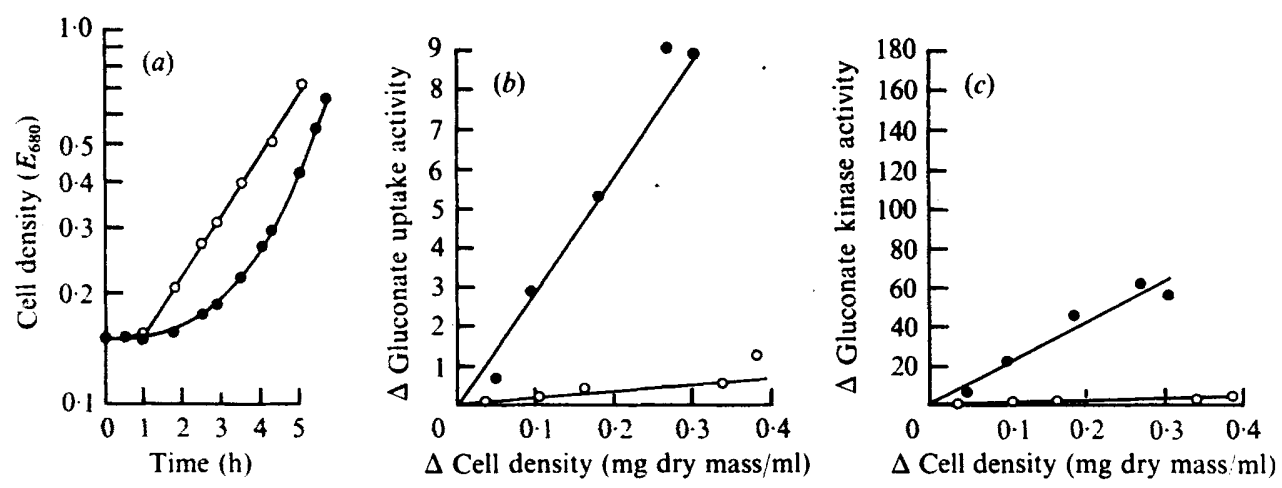

Fig. 7. The growth $(a)$, the uptake of gluconate $(b)$, and the activity of gluconate kınase $(c)$, of fructose-grown strain BBI38 (GntM- $\mathbf{G n t S}^{+}$) adapting to growth on $(\bullet)$ Io mM-gluconate or $(O)$ Io mM-gluconate + $10 \mathrm{~mm}$ pyruvate. Samples were treated as described for Fig. 3. 
Table 4. Maintenance of induction of gluconate uptake and gluconate kinase in strain $\mathrm{BBI} 38$ (wild-type phenotype) during growth on different carbon sources

Cultures of strain BBI 38 were grown on 10 mM-gluconate overnight, harvested and resuspended in fresh medium containing $10 \mathrm{mM}$-gluconate $+20 \mathrm{mM}$ of a different carbon source as listed below. The cells were grown for two generations and their gluconate uptake and gluconate kinase activities determined as described in Methods.

$\begin{array}{ccc}\text { Carbon source } & \text { Gluconate uptake } & \text { Gluconate kinase } \\ \text { Gluconate } & 31 & 690 \\ \text { + Pyruvate } & 4 & 50 \\ \text { + Fructose } & 5 & 120 \\ \text { + Fumarate } & 7 & 160 \\ \text { + Succinate } & 6 & 160 \\ \text { + Proline } & 24 & 440 \\ \text { + Acetate } & 15 & 470\end{array}$

acetate, in the growth medium. Gluconate kinase was similarly repressed by these materials; however, the effect of pyruvate on the synthesis of this enzyme was even more marked than its effect on the formation of the gluconate uptake system. Since the predominant route for the catabolism of gluconate in E. coli is via the Entner-Doudoroff pathway (Zablotny \& Fraenkel, I967) that yields I molecule of glyceraldehyde-3-phosphate and I molecule of pyruvate for each molecule of gluconate that enters this sequence, a role of pyruvate as a regulator of the synthesis of the proteins that catalyse the initial steps of gluconate catabolism would be analogous to the action of other metabolic 'feed-back' systems that operate at the level of protein synthesis.

In contrast to the striking repression of the protein(s) specified by the $g n t S^{+}$marker, we have not found any significant inhibition by pyruvate of the activity of the gluconate uptake system in wild-type organisms, or in mutants that lack either functional gnt $R^{+}$or $g n t S^{+}$ genes.

\section{Properties of gluconate ${ }^{+}$revertants of the $\mathrm{GntM}^{-} \mathrm{GntS}^{-}$mutant BBI}

Colonies of strain BBI are red on gluconate-tetrazolium plates and show no growth on plates containing gluconate as sole carbon source, but readily give rise to progeny able to grow on gluconate, with a high mutation rate of about $0.2 \times 10^{-6}$ per bacterial division. Over 500 independent gluconate ${ }^{+}$organisms, obtained by incubating single-colony isolates of strain BBI for 3 days at $37^{\circ} \mathrm{C}$ on plates containing gluconate as sole carbon source, were found to be white on gluconate-tetrazolium plates, indicating reversion of $\mathrm{GntM}^{-}$to GntM $^{+}$.

Ten such independent phenotypic revertants were tested for the inducibility of the gluconate uptake system and of gluconate kinase. Six of these were found to be inducible for both properties. After growth on Io mM-fructose, these revertants on average took up I $\mathrm{nmol}$ of gluconate $/ \mathrm{min} / \mathrm{mg}$ dry mass, and sonic extracts contained gluconate kinase activity sufficient to convert about II nmol of gluconate to 6-phosphogluconate/min/mg protein; after two doublings on gluconate growth medium, these activities rose to average values of 18 and 400 , respectively. However, the four remaining revertants analysed had become constitutive for both gluconate uptake and gluconate kinase. The average gluconate uptake activity after growth on fructose or gluconate was 25 or $18 \mathrm{nmol} / \mathrm{min} / \mathrm{mg}$ dry mass, respectively, whereas the gluconate kinase activity for either growth condition was about $900 \mathrm{nmol} / \mathrm{min} / \mathrm{mg}$ protein, which is double the activity observed in extracts of fully-induced wild-type strains or inducible gluconate ${ }^{+}$revertants. 
One of these constitutive revertants, strain BBI2, was further analysed in two ways. Extracts of this organism, grown on glycerol, were found to contain 6-phosphogluconate dehydratase activity at $2850 \mathrm{nmol} / \mathrm{min} / \mathrm{mg}$ protein. This was even higher than the activity observed with extracts of gluconate-grown wild-type cells (Kornberg \& Soutar, 1973) and shows that this enzyme had also become constitutive. Whether this mutant still carried the gnt $S$ allele present in its parent strain BBI was tested by growing phage PI on it and transducing this phage into the GntM- GntS ${ }^{+}$strain BB54 that also carried the marker $f d p$; Fdp + transductants were selected and tested for growth on gluconate. Twenty-one out of 9I transductants thus obtained were now totally unable to grow on gluconate, which showed that the phage grown on the constitutive strain had brought the $\mathrm{GntS}^{-}$phenotype into the already GntM- recipient to over $20 \%$, concomitantly with $f d p^{+}$. In another test, recombinants were obtained from crosses of the constitutive mutant BBI 2 with the Hfr strain PIO, and were selected for $\mathrm{Mal}^{+} \mathrm{Str}^{\mathrm{R}}$. Analysis of such recombinants suggested that the mutation responsible for constitutivity maps near malA, since 3 out of 4 recombinants selected for $\mathrm{Mal}^{+}$had become inducible. All these results confirm the finding by Zwaig et al. (1973) that $E$. coli contains a regulatory gene $\left(g n t R^{+}\right)$for gluconate uptake, gluconate kinase and 6phosphogluconate dehydratase, and that mutation of this gene can occur in at least two ways. Change of gnt $R^{+}$to gnt $R$ I leads to the $\mathrm{GntM}^{-}$phenotype, in which the three activities are inducible to only a small extent and with difficulty; on the other hand, mutation of $g n t R^{+}$to $g n t R$ leads to derepression of these activities.

There are two main models that have been postulated to account for phenotypes of the

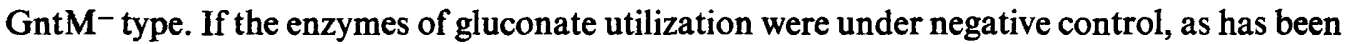
established for the lac operon, the $\mathrm{GntM}^{-}$phenotype would be akin to that exhibited by 'super-repressed' $i^{i}$ mutants (Willson et al. 1964). If this were so, gntR I mutants might contain an altered repressor incapable of recognizing the normal inducer; further mutations within the gnt $R$ gene would then be expected to alter the site that binds to the operator (leading to constitutivity), or to revert the original lesion to $g n t R^{+}$(and thus leading to the restoration of the $\mathrm{GntM}^{+}$phenotype). In contrast, if the enzymes of gluconate utilization were under positive control, as has been established for the enzymes of arabinose utilization (Englesberg, Squires \& Meronk, 1969), gnt $R^{+}$would code for a regulatory protein that might act as a repressor in the absence of gluconate, but would activate the structural genes for gluconate uptake, kinase and dehydratase in its presence. Mutants of the GntM- phenotype would be likely not to produce the required product of the $g n t R^{+}$gene, or to produce it in a form unable to bind gluconate, or to be unable, for one of several reasons, to activate the structural genes for gluconate utilization. Mutants constitutive for these three activities would presumably form a regulatory gene product that is an activator even in the absence of gluconate.

It is not yet possible to distinguish unequivocally between these positive and negative control models, but we tend to favour the negative model for several reasons:

(i) Mutation of 'super-repressed' gntRI mutants to $g n t R^{+}$(wild-type) on the one hand, and to gnt $R$ (constitutive) on the other, might be expected to occur with roughly equal frequency. However, mutation of organisms unable to form a regulatory gene product, or unable to bind gluconate, to a form in which the regulatory protein is formed in an active gluconate-independent configuration, would be likely to occur much less frequently than reversion to the wild-type phenotype. It will be recalled that four out of ten GntM+ revertants of the GntM- mutant BBI were constitutive, whereas the other six exhibited the wildtype character.

(ii) A further class of revertant of BBI grew on gluconate at $40^{\circ} \mathrm{C}$ but not at $22{ }^{\circ} \mathrm{C}$. Cells 
Table 5. Gluconate uptake and gluconate kinase in constitutive and diploid strains of E. coli

The constitutive (gntR) strain BB77, and merozygotes carrying either the episome $\mathrm{F}^{\prime} \mathrm{I} 40$ or $\mathrm{F}^{\prime} \mathrm{I}$ II, were grown on $10 \mathrm{mM}$-fructose or $5 \mathrm{mM}$-maltose overnight, harvested and grown on $10 \mathrm{~mm}$-fructose, or $5 \mathrm{~mm}$-maltose, or $10 \mathrm{~mm}$-gluconate for two doublings. Gluconate kinase and gluconate uptake were determined as described in Methods. 'Cured' strains were colonies isolated from the diploids, which had lost the episome spontaneously. These, and the constitutive strain BB77, did not grow on maltose and were grown only on $10 \mathrm{~mm}$-fructose.

\begin{tabular}{|c|c|c|c|c|c|}
\hline \multirow{3}{*}{ Description } & \multirow{3}{*}{ Strain } & \multicolumn{4}{|c|}{ Specific activity of } \\
\hline & & \multicolumn{2}{|c|}{ Gluconate uptake on } & \multicolumn{2}{|c|}{ Gluconate kinase on } \\
\hline & & $\begin{array}{l}\text { Maltose } \\
\text { or fructose }\end{array}$ & Gluconate & $\begin{array}{l}\text { Maltose } \\
\text { or fructose }\end{array}$ & Gluconate \\
\hline gnt $R$ & BB77 & $3 \mathrm{I}$ & 17 & II 50 & 493 \\
\hline$F^{\prime} 140-g n t R^{+} / g n t R$ & $\begin{array}{l}\text { Diploid I } \\
\text { Diploid } 2\end{array}$ & $\begin{array}{l}0.6 \\
-\end{array}$ & $\begin{array}{l}\text { II } \\
\text { I3 }\end{array}$ & $\begin{array}{r}7 \\
13\end{array}$ & $\begin{array}{l}520 \\
553\end{array}$ \\
\hline$F^{\prime} 14 \mathrm{I}-g n t R^{+} / g n t R$ & $\begin{array}{l}\text { Diploid } 3 \\
\text { Diploid } 4\end{array}$ & $\begin{array}{l}0.3 \\
0.6\end{array}$ & $\begin{array}{l}17 \\
17\end{array}$ & $\begin{array}{r}4 \\
12\end{array}$ & $\begin{array}{l}478 \\
520\end{array}$ \\
\hline$g n t R$ & $\begin{array}{l}\text { Cured I } \\
\text { Cured } 2 \\
\text { Cured } 3 \\
\text { Cured } 4\end{array}$ & $\begin{array}{l}26 \\
35 \\
35 \\
23\end{array}$ & $\begin{array}{c}- \\
- \\
-\end{array}$ & $\frac{940}{-}$ & $\begin{array}{l}- \\
-\end{array}$ \\
\hline
\end{tabular}

cultured at $22{ }^{\circ} \mathrm{C}$ took up gluconate at less than I $\mathrm{nmol} / \mathrm{min} / \mathrm{mg}$ dry mass, even if grown on glycerol, fructose or nutrient broth in the presence of gluconate; however, they took up gluconate at 9 to $21 \mathrm{nmol} / \mathrm{min} / \mathrm{mg}$ dry mass after growth at $37^{\circ} \mathrm{C}$ on these carbon sources even in the absence of gluconate. As with other constitutive revertants of strain BBI, the lesion in these temperature-sensitive constitutive mutants appeared to be linked to the malA region. This behaviour is readily explained as the consequence of an alteration of a site on a repressor that renders it unable to bind to the operator at $40^{\circ} \mathrm{C}$ though it still binds at $22{ }^{\circ} \mathrm{C}$, leading to the temperature-dependent constitutivity observed. On the basis of a positive control model, mutants of this type would have to contain a regulatory protein that acts as activator at $40^{\circ} \mathrm{C}$ in the absence of gluconate but that, at $22{ }^{\circ} \mathrm{C}$, acts solely as repressor even in the presence of gluconate.

(iii) By means of $\mathrm{F}^{\prime}$ factors carrying the ara region, the dominance relationships of the various araC alleles have been shown to be quite different from those of the phenotypically similar $i^{s}$ mutants of the lac operon. In particular, $i^{s}$ mutations, which lead to non-inducible 'switching off' of the lac operon, are dominant in trans to $i^{+}$(inducible) and $i^{-}$(constitutive) mutations since the cytoplasmic super-repressor is postulated not to be inactivated by inducer. On the contrary, ara $C^{-}$mutations are recessive in trans to both $C^{+}$(inducible) in the presence of arabinose, and to $C^{\circ}$ (constitutive) in its absence: this latter behaviour is because the $a r a C$ gene product in $C^{\circ}$ mutants has been so altered that it activates the structural genes of arabinose utilization under all circumstances.

Table 5 records the behaviour of merozygotes in which $F^{\prime}$ episomes derived from two $E$. coli strains inducible for gluconate uptake and for gluconate kinase were introduced into the recipient strain BB77 that was constitutive and carried the markers gntS as well as malA and $\operatorname{rec} A$. All diploids tested had become inducible for both uptake and phosphorylation of gluconate; on eliminating the $F^{\prime}$ episomes from four of these merozygotes, the resultant 'cured' strains regained the $\mathrm{Mal}^{-}$phenotype, and the constitutivity of the uptake system and of gluconate kinase. These results show that the allele(s) that determines inducibility is dominant to that specifying constitutivity, as expected from the negative control envisaged. 
In an analogous experiment, the same $\mathrm{F}^{\prime}$ episomes derived from the inducible strains of $E$. coli were introduced into a recipient strain which carried the $g n t R I$ gntS markers as well as malA and $\operatorname{rec} A$. The resultant partial diploids showed only background growth on gluconate plates, and grew with a mean doubling time of nearly $4 \mathrm{~h}$ on liquid medium containing gluconate as sole carbon source. Cells harvested from this medium took up only Io nmol of gluconate $/ \mathrm{min} / \mathrm{mg}$ dry mass, and extracts of these cells contained gluconate kinase at specific activity of only $60 \mathrm{nmol} / \mathrm{min} / \mathrm{mg}$ protein. This behaviour is again consistent with the view that the GntM- phenotype is the result of an $i^{\mathrm{s}}$-type mutation. In the present instance, the merozygotes would contain both the gene that specifies the super-repressor and the gene that specifies the wild-type product: as a result, the diploid would be less easily induced to form the uptake system and the kinase than would $g n t R^{+}$cells, but would be more inducible than gntRI mutants.

The observation that in $\mathrm{GntM}^{-} \mathrm{GntS}+$ mutants the gluconate uptake system can be fully, and gluconate kinase partially, induced further suggests that the regulatory gene specified by $g n t R^{+}$is different from that governing the expression of the gntS region; the initial steps of gluconate catabolism thus appear to be duplicated by genes located in two regulatory units some 20 min apart on the linkage-map. The striking effects of pyruvate in almost totally preventing the expression of the gnt $S^{+}$gene(s), whilst having only a slight effect on those controlled by gnt $R^{+}$, further distinguish these two regulatory units. This duality of control is also in accord with reports that $E$. coli contain two forms of gluconate kinase (Hung et al. 1970) and two gluconate uptake systems (Nagel de Zwaig et al. 1973).

We thank Miss Elizabeth Hill for technical assistance, Dr Maurice Jones-Mortimer for many helpful discussions, Drs Barbara Bachmann (E. coli Genetic Stock Center), R. A. Cooper (Leicester University) and F. Jacob (Institut Pasteur) for generous gifts of $E$. coli strains, and the Science Research Council for support under grant B/SR/72462.

\section{REFERENCES}

BÄCHI, B. \& Kornerg, H. L. (1975) Utilization of gluconate by Escherichia coli. A role of adenosine $3^{\prime}, 5^{\prime}$-cyclic monophosphate in the induction of gluconate catabolism. Biochemical Journal 150, $123-128$.

Brice, C. B. \& Kornberg, H. L. (I967). Location of a gene specifying phosphopyruvate synthase activity on the genome of Escherichia coli, KI 2. Proceedings of the Royal Society B 168, 28I-292.

COHEN, S. S. (1951). Gluconokinase and the oxidative path of glucose-6-phosphate utilization. Journal of Biological Chemistry 189, 617-628.

Eisenberg, R. C. \& Dobrogosz, W. J. (1967). Gluconate metabolism in Escherichia coli. Journal of Bacteriology $93,94 \mathrm{I}-949$.

Englesberg, E., SQuires, C. \& Meronk, F. (1969). The L-arabinose operon in Escherichia coli $\mathrm{B} / \mathrm{r}$ : a genetic demonstration of two functional states of the product of a regulator gene. Proceedings of the National Academy of Sciences of the United States of America 62, $1100-1107$.

ESSENBERG, R. C. \& KornberG, H. L. (1975). Energy coupling in the uptake of hexose phosphates by Escherichia coli. Journal of Biological Chemistry 250, 939-945.

FAIK, P. \& KoRNBERG, H. L. (1973). Isolation and properties of $E$. coli mutants affected in gluconate uptake. FEBS Letters 32, 260-264.

Fraenkel, D. G. \& Banerjee, S. (1972). Deletion mapping of $z w f$, the gene for a constitutive enzyme, glucose 6-phosphate dehydrogenase, in Escherichia coli. Genetics 7r, 481-489.

Hung, A., Orozco, A. \& ZwaIG, N. (1970). Evidence for two gluconokinase activities in Escherichia coli. Bacteriological Proceedings, Abstract, p. 148.

Kornberg, H. L. \& Smith, J. (1969). Genetic control of hexose phosphate uptake by Escherichia coli. Nature, London 224, 1261-1 262. 
KorNBerg, H. L. \& SoutAR, A. K. (1973). Utilization of gluconate by Escherichia coli. Induction of gluconate kinase and 6-phosphogluconate dehydratase activities. Biochemical Journal 134, 489-498.

MilleR, J. H. (1972). Experiments in molecular genetics. New York: Cold Spring Harbor Laboratory.

MoNOD, J. (1956). Remarks on the mechanism of enzyme induction. In Enzymes: Units of Biological Structure and Function, pp. 7-28. Edited by O. H. Gaebler. New York: Academic Press.

MoRgAN, M. J. \& KoRNBERG, H. L. (I969). Regulation of sugar accumulation by Escherichia coli. FEBS Letters 3, 53-56.

Nagel De Zwaig, R., Zwaig, N., Istúriz, T. \& SanChez, R. S. (1973). Mutations affecting gluconate metabolism in Escherichia coli. Journal of Bacteriology II4, 463-468.

POUYSSÉGUR, J. M., FAIK, P. \& KoRNBERG, H. L. (1974). Utilization of gluconate by Escherichia coli. Uptake of D-gluconate by a mutant impaired in gluconate kinase activity and by membrane vesicles derived therefrom. Biochemical Journal r40, 193-203.

RoBIN, A. \& KEPES, A. (1973). The mechanism of maintenance of electroneutrality during the transport of gluconate by $E$. coli. FEBS Letters 36, 133-1 36.

Roseman, S. (I969). The transport of carbohydrates by a bacterial phosphotransferase system. Journal of General Physiology 54, 1385-1 $80 \mathrm{~s}$.

TAYLOR, A. L. \& TROTTER, C. D. (1972). Linkage map of Escherichia coli sträin K-I 2. Bacteriological Reviews 36, 504-524.

Willson, C., Perrin, D., Cohn, M., JACoB, F. \& Monod, J. (1964). Non-inducible mutants of the regulator gene in the 'lactose' system of Escherichia coli. Journal of Molecular Biology 8, 582-592.

YU, M. T., KANEY, A. R. \& ATwOoD, K. C. (1965). Genetic mapping of fructose-I,6-diphosphatase in Escherichia coli. Journal of Bacteriology 90, $1150-1152$.

ZABLOTNY, R. \& FrAENKEL, D. G. (1967). Glucose and gluconate metabolism in a mutant of Escherichia coli lacking gluconate-6-phosphate dehydrase. Journal of Bacteriology 93, 1579-1 581.

Zwaig, N., NAGEL DE ZWAig, R., Istúriz, T. \& WeCKSLER, M. (1973). Regulatory mutations affecting the gluconate system in Escherichia coli. Journal of Bacteriology I14, 469-473. 